Research Article

\title{
Self-Organized Criticality and Trend Analysis in Time Series of Blackouts for the China Power Grid
}

\author{
Qun Yu $\mathbb{D}^{1}{ }^{1} \mathrm{Na}$ Cao, ${ }^{1}$ Qilin Liu, ${ }^{1}$ Yuqing Qu, ${ }^{2}$ and Yumin Zhang $\mathbb{D}^{1}$ \\ ${ }^{1}$ School of Electrical Engineering and Automation, Shandong University of Science and Technology, Qingdao 266590, China \\ ${ }^{2}$ Key Laboratory of the Ministry of Education on Smart Power Grids, Tianjin University, Tianjin 300072, China \\ Correspondence should be addressed to Qun Yu; yuqun_70@163.com
}

Received 6 June 2020; Revised 4 August 2020; Accepted 10 August 2020; Published 29 August 2020

Guest Editor: Zaoli Yang

Copyright (C) 2020 Qun Yu et al. This is an open access article distributed under the Creative Commons Attribution License, which permits unrestricted use, distribution, and reproduction in any medium, provided the original work is properly cited.

\begin{abstract}
This paper proposes effective evidence on the correlation between trend and self-organized criticality (SOC) of the power outage sequence in China. Taking the data series of blackouts from 1981 to 2014 in the China power grid as the research object, the method of $V / S$ is introduced into the analysis of the power system blackout sequence to demonstrate their prominent long-time correlations. It also verifies the probability distribution of load loss about blackout size in the China power grid has a tail feature, which shows that the time series of blackouts in the China power grid is consistent with SOC. Meanwhile, a kind of mathematical statistics analysis is presented to prove that there is a seasonal trend of blackouts, and the blackout frequency and blackout size have not decreased over time but have an upward trend in the China power grid, thereby indicating that blackout risk may be increasing with time. The last 34 years' data samples of power failure accidents in the China power grid are used to test the proposed method, and the numerical results show that the proposed self-organized criticality and trend analysis method can pave the way for further exploration of the mechanism of power failure in the China power grid.
\end{abstract}

\section{Introduction}

In contemporary society, electricity has become an essential energy for the development of all countries in the world, and the safe operation of the power grid is very important. However, domestic and foreign power grid blackouts occur frequently, which brought great impact to all countries. For example, in 2003, the large blackout in the east and Canada of the United States caused 61.8 GW load damage and \$30 billion loss. In 2009, the large blackout in Brazil resulted in $24 \mathrm{GW}$ load loss. In 2011, the large blackout in Japan brought about $22 \mathrm{GW}$ load loss. In 2012, the large blackout in India led to $50 \mathrm{GW}$ load damage. These large blackouts often begin from a single component failure in the system, causing cascading failure in turn. To study the internal dynamic mechanism of blackouts for preventing the occurrence of large blackouts and to reduce the adverse effects and losses caused by blackouts, domestic and foreign scholars have done a lot of research and analysis on power system blackouts.
Twenty years of blackout data in the China power grid from 1981 to 2000 inclusive were analyzed by Guo et al. [1-3]. They used mathematical statistics to analyze the blackouts in the China power grid and obtained some conclusions. They observed an upward trend in blackout size and a downward trend in blackout frequency. Besides, it has been found that due to natural disasters, the number of blackouts caused by mechanical failures and human error is on the rise. Twenty-two years' data from 1981 to 2002 were analyzed by Yu and Guo [4-7]. They found that blackout size follows a power-law probability distribution in the China power grid. Duan and Su [8] analyzed the long-term correlation and power-law distribution of grid faults and revealed the self-organized criticality of fault time series of transmission systems or distribution systems. Chao et al. [9] analyzed a time series of blackouts in the Puyang power grid and calculated the Hurst exponent [10]. They found that the probability distribution of the number of faults per day follows the power law, and the Hurst exponent values were around 0.67 , indicating long-term correlation, so the faults 
occurred in Puyang distribution network possessed SOC. Xu et al. [11] analyzed the distribution of times between faults in the Guangdong power grid from 2000 to 2013 inclusive. They found that the probability distribution of time between faults has a power-law tail. They used rescaled range analysis and scaled windowed variance to calculate the Hurst exponent and found values close to 1.0, indicating long-term correlation and self-similarity. Yu et al. [12] combined the method of relative value and the method of $R / S$ to analyze the correlation of the load loss sequence of the China power grid from 1981 to 2014, pointing out that the positive correlation of the loss load of the China power grid blackout is getting stronger. From a macro point of view, the number of loss of the China power grid in the next few years will increase, but the relative value of the loss load will decrease. The time interval series of blackout accidents in the China power grid also have long-range positive correlation and statistical self-similarity, which were obtained by $\mathrm{Yu}$ et al. [13] using the method of $V / S$.

Carreras et al. [14] analyzed the four-year data of North American power systems from 1994 to 1997 and calculated that the Hurst exponent of blackout size was greater than 0.5 , indicating long-range correlation and self-similarity. Subsequently, Carreras et al. [15] analyzed the 15-year data from 1984 to 1998 . They found that the probability distribution of blackout size has a power-law tail and has a long-term correlation. They also found that the results from a sand pile model, known to be SOC, agree with the disturbance data, following the work of Bak et al. [16]. Thus, they considered that the blackout data seem consistent with SOC. They also raised that there may be seasonal periodicities in blackout data, but no evidence was found. Simonoff et al. [17] analyzed 14 years of blackout data from 1990 to 2004 of the North American power system. They used statistical hypothesis testing to enhance the credibility of conclusions. They found some new conclusions that the number of blackouts in summer is higher than that in winter, and the number of blackouts tends to increase by $14 \%$ of the year. They also found that, as weather events became more common, equipment failures will be less. Possible trends or changes in blackouts over time were analyzed by Amin and Hines et al. [18, 19]. Hines et al. [19] analyzed 23 years' data of North American power system blackouts from 1984 to 2006 inclusive. They described several patterns that appear in the data. These included the observation that the number of large blackouts has not decreased, blackouts show seasonal trends and time-of-day trends, and there is no apparent positive correlation between blackout size and restoration time. Seasonal variations were analyzed in $[20,21]$. Cornforth [21] found the long-tail distribution of the 23-year electrical disturbance data. They also found a seasonal trend in disturbances, with an increasing number of disturbances and severity of disturbances measured by load loss and by customers affected. Carreras et al. [22] analyzed 22 years' data of North American electric power system blackouts from 1984 to 2006 . They validated the power law and long-term correlation of the East-West interconnection blackouts in North American power grid and analyzed the waiting time distribution. Besides, they found that the annual mean blackout size is highly variable, and the risk of large blackouts is greater than that of medium size.

The work reported here is based on the 34-year blackout data in the China power grid from 1981 to 2014. Based on this relatively complete dataset, we analyze the SOC and possible trends of blackouts in the China power grid. The results observed in this paper can provide reference for effective blackout-risk assessment, and it is of great significance to guide the planning and construction of the power system.

The major contributions of this paper are summarized as follows:

(1) This paper proposes effective evidence on the correlation between trend and self-organized criticality (SOC) of the power outage sequence in China

(2) The $V / S$ method is proposed to demonstrate that the trend and self-organized criticality (SOC) of the power outage sequence have prominent long-time correlations

(3) A kind of mathematical statistics analysis is presented to prove the law of power blackouts in the China power grid, which will have an important implication for the investment in the electricity industry

The rest of this paper is organized as follows. Section 2 introduces the dataset and research analysis method. Section 3 investigates the time series of blackouts based on selforganized criticality (SOC). Section 4 analyzes the trends of time series of blackouts. The conclusions are drawn in Section 5 .

\section{The Blackout Data in China from 1981 to 2014 and the Methodology}

2.1. The Dataset. We analyzed the 34 -year blackout data in the China power grid from 1981 to 2014, which are available publicly from [23-32]. Some blackouts have the record of load loss, and some do not have. To make the analysis results reliable and accurate, we selected the 277 blackouts with load loss record as the research object, and other blackouts without load loss record were filtered out. On average, 8.15 blackouts occurred in a year, indicating that a blackout occurred every 44.6 days. The average load loss of blackout size per year was $3518.2 \mathrm{MW}$, and the average load loss per blackout per year was 431.7 MW. Besides, we conducted a statistical analysis of blackouts with the load loss greater than $300 \mathrm{MW}$. Figure 1 shows a time series of blackouts in the China power grid.

2.2. The Methodology. The blackout data were imported to Excel; manually record and review each record repeatedly to ensure accuracy. In order to investigate SOC of time series of blackouts, we used $V / S$ to analyze the correlation of the time series of blackouts in the China power grid and used Clauset's method suggested by Clauset et al. [33] to analyze the power law of load loss about blackout size in the China power grid. To test the upward trend in blackout frequency 


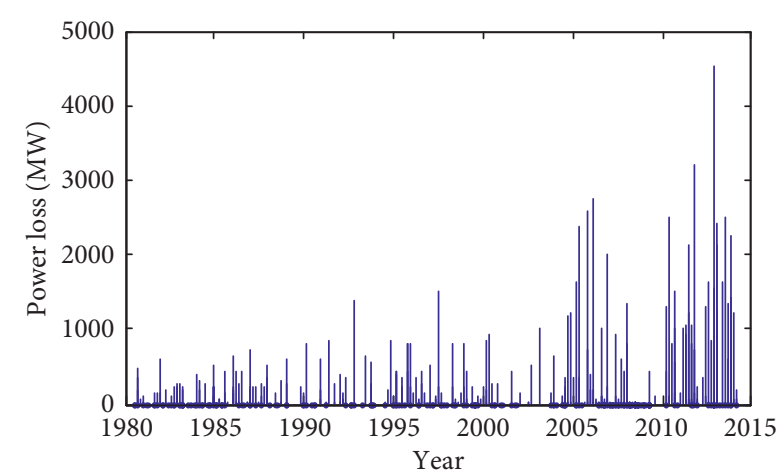

Figure 1: Time series of blackouts in the China power grid from 1981 to 2014.

and blackout size, we divided the data into 34 groups yearly, corresponding to the year in which they were reported. The number and load loss maps of annual blackout were plotted. To test the seasonal trends in the blackout data, we divided the data into 12 groups every month, corresponding to the months they were reported. From the data of the 34-year blackout event, the seasonal trend is obvious. However, to test this result, we compared the summer data with the data from the entire dataset and calculated the 95\% confidence interval to assess the importance of this difference.

\section{SOC of Time Series of Blackouts in the China Power Grid}

3.1. Long-Time Correlation. If a power-law curve progressively attenuates the autocorrelation function of a time series, the time series can be considered to have a long-time correlation [15]. $\mathrm{V} / \mathrm{S}$ is used to calculate the Hurst exponent [34] to determine the long-range positive correlation of blackouts in the China power grid. The $V / S$ method can be explained as follows [35].

$\bar{x}$ is the sample mean $(1 / n) \sum_{t=1}^{n} x_{t}$, and $S_{n, q}^{2}$ is an estimator of $\sigma^{2}=\sum_{\mathrm{t}} \operatorname{Cov}\left(x_{t}, x_{0}\right)$ defined as

$$
S_{n, q}^{2}=\frac{1}{n} \sum_{t=1}^{n}\left(x_{t}-\bar{x}\right)^{2}+2 \sum_{t=1}^{q} w_{t}(q) \gamma_{t},
$$

where $n$ is the number of the time series.

In (1),

$$
w_{t}(q)=1-\frac{t}{q+1}, \quad q<n,
$$

$w_{t}(q)$ are the Bartlett weights, and $\gamma_{t}$ are the sample covariance:

$$
\gamma_{t}=\frac{\sum_{i=1}^{n-t}\left(x_{i}-\bar{x}\right)\left(x_{i+t}-\bar{x}\right)}{n}, \quad 0 \leq t<n .
$$

Then, the statistic $V_{s}(n)$ is defined as follows:

$$
V_{s}(n)=\frac{\left[\sum_{k=1}^{n}\left(\sum_{t=1}^{k}\left(x_{t}-\bar{x}\right)\right)^{2}-(1 / n)\left(\sum_{k=1}^{n} \sum_{t=1}^{k}\left(x_{t}-\bar{x}\right)\right)^{2}\right]}{n^{2} S_{n, q}^{2}} \text {. }
$$

The above process is repeated for every scale, $n, 2 n, 3 n$, $\ldots, k^{*} n$. The scaling relationship is

$$
V_{s}(n) \approx c \cdot n^{2 H}
$$

Taking the logarithm of both ends of equation (5),

$$
\lg \left[V_{s}(n)\right]=\lg c+2 H \lg n,
$$

where $c$ is the statistical constant and $H$ is the Hurst exponent.

The correlation can be judged based on the Hurst exponent of the time series. When $H=0.5$, it indicates that the time series is uncorrelated and random. When $0.5<H<1.0$, it indicates that the time series has long-range time correlations. When $0<H<0.5$, it indicates that the time series has long-range anticorrelations.

We used the $V / S$ method to analyze the long-range correlation of time series about blackouts in the China power grid. The statistical results are shown in Figure 2. From Figure 2, we can see that the $V_{s}(n)$ statistics of the China power grid fit well with the fit line. The Hurst exponent of the load loss of blackout size in the China power grid is 0.7, which indicates that there is a significant long-range time correlation in time series of blackouts in the China power grid.

3.2. Probability Distribution. In order to make the analysis result reasonable and effective and to avoid the least square fitting method to analyze the data power-law deviation, we used the method described in [34] to analyze and test the power law of blackouts in the China power grid. Its powerlaw model parameters are shown in Table 1, and its cumulative probability distribution is shown in Figure 3.

It is known from Table 2 that $p>0.1$, according to which it can be considered that the power-law distribution of load loss about blackout size in the China power grid is reasonable. Also, it is possible to exclude the hypothesis that other distributions, such as Poisson or exponential distribution, are more suitable than power-law distributions. The logarithmic likelihood ratios (LR) of the powerlaw distribution to the Poisson distribution and the power-law distribution to the exponential distribution are calculated by likelihood ratio test. The results are shown in Table 2.

From Table 2, we can see that the LR of the power-law distribution and Poisson distribution and exponential distribution are both greater than 0 , indicating that the power-law distribution is more suitable, and the Poisson distribution and the exponential distribution have $p$ less than 0.1 , which can reject the hypothesis that the load loss about blackout size in the China power grid follows the Poisson distribution or exponential distribution.

Based on the above analysis, it can be seen that the load loss about blackout size in the China power grid does not satisfy the Poisson distribution or the exponential distribution but approximates the power-law distribution, which strongly indicates that the time series of blackouts in the China power grid has the characteristic of SOC. 


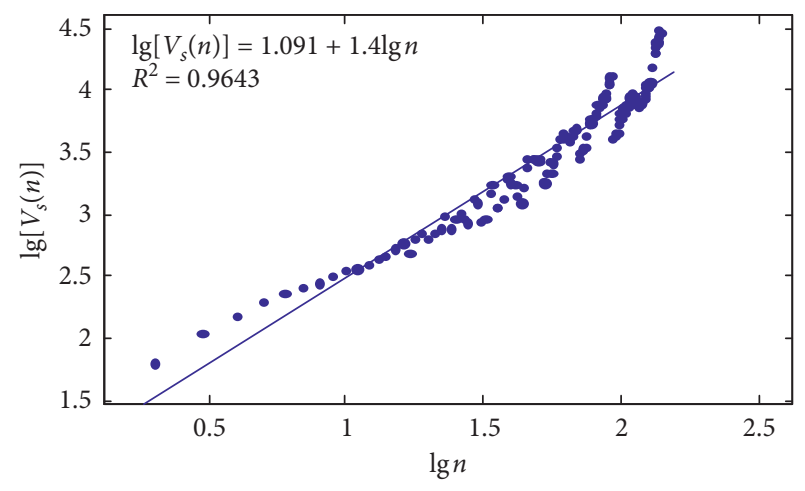

Figure 2: Log-log plot of $V_{s}(n)$ and $n$ of blackouts in the China power grid.

TABle 1: Power-law model parameters of load loss about blackout size in the China power grid.

\begin{tabular}{lcccc}
\hline$n^{1}$ & $x_{\min }{ }^{2}$ & $\alpha^{3}$ & $n_{\text {tail }}{ }^{4}$ & $p^{5}$ \\
\hline 277 & 737 & 2.86 & 52 & 0.15 \\
\hline
\end{tabular}

${ }^{1} n$ is the number of samples. ${ }^{2} x_{\min }$ is the lower bound of the power-law behavior. ${ }^{3} \alpha$ is the estimated value of the power exponent. ${ }^{4} n_{\text {tail }}$ is the standard deviation of the uncertainty of the fitting parameter. ${ }^{5} p$ is the rationality of the quantified power-law hypothesis; when $p>0.1$, indicating that the power-law hypothesis is reasonable, there is a reason to believe that the data satisfy the power-law distribution. When $p \leq 0.1$, the power-law hypothesis is rejected, and the data do not satisfy the power-law distribution.

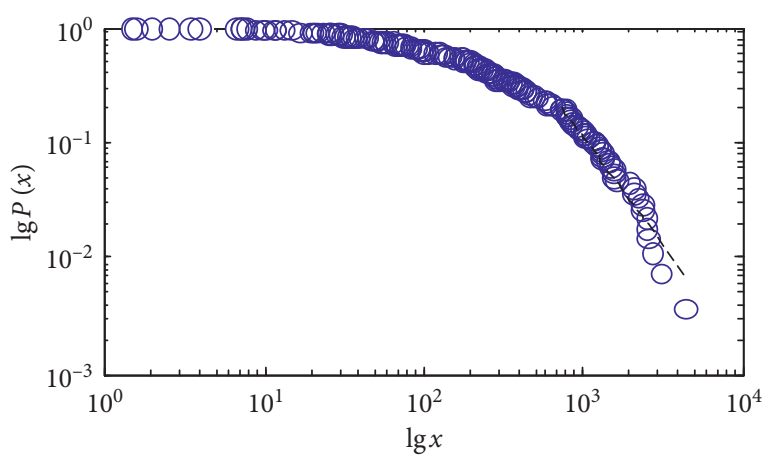

FIGURE 3: Log-log plot of load loss about blackout size in the China power grid.

TABLE 2: Log-likelihood ratio test of the power-law distribution to the Poisson distribution and exponential distribution.

\begin{tabular}{lccccc}
\hline \multicolumn{2}{c}{ Power law } & \multicolumn{2}{c}{ Poisson } & \multicolumn{2}{c}{ Exponential } \\
\hline LR & $p$ & LR & $p$ & LR & $p$ \\
5.29 & 0.15 & 6.31 & 0.04 & 4.26 & 0.08 \\
\hline
\end{tabular}

\section{Trends in the Blackout in the China Power Grid}

4.1. The Trend of Blackout Frequency and Blackout Size. Figure 4 shows the number of blackouts per year in the China power grid, and the magnitude of these blackouts varies in size (measured in MW). The number of blackouts

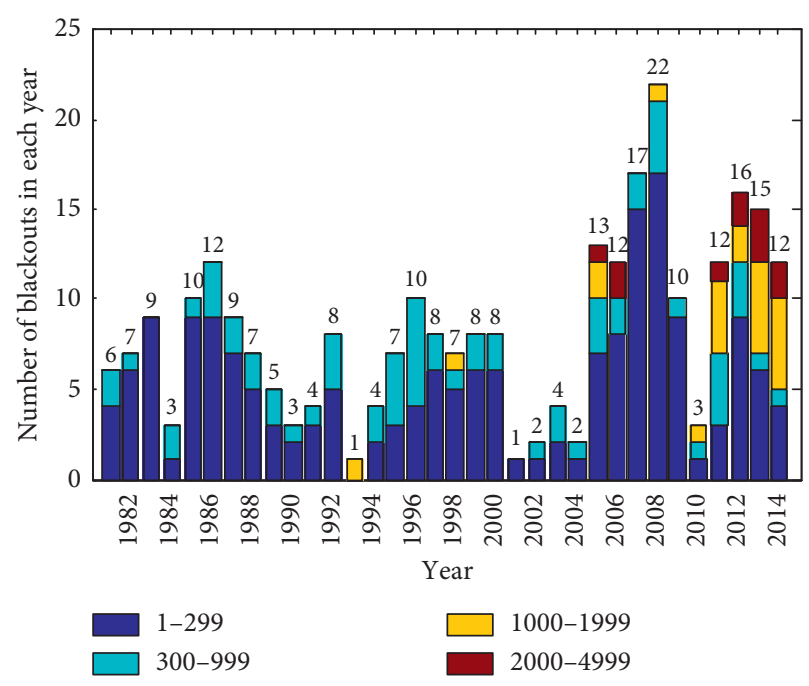

FIGURE 4: The number of blackouts per year in the China power grid.

per year in the China power grid has no obvious periodicity and has no clear increase or decrease trend. However, beginning in 2005, except for 2010, the number of blackouts per year is greater than the average number of 8.15 of blackouts per year, which indicates that the blackout frequency has not decreased and has the possibility of an upward trend over time in the China power grid. Besides, 22 blackouts occurred in 2008, related to the extremely rainy and snowy weather in the South China power grid for a long time.

Figure 5 shows the load loss of blackout size per year for all 277 event records. The year with the greatest load loss of blackout size is 2013, which is related to a major cascading failure. The large blackouts of 2005, 2006, 2007, 2011, 2012, and 2014 are also apparent. Beginning in 2005, except for 2009 and 2010, the load loss of blackout size per year is greater than the average of 3,517.3 MW, which indicates that the load loss of blackout size has not decreased over time in the China power grid and has the possibility of an upward trend.

Figures 4 and 5 show that the number of blackouts and the load loss of blackout size per year in the China power grid have not decreased over time and have the possibility of an upward trend. To test our conclusions, we divided the 34 years' data into six periods. Table 3 shows some descriptive statistics for these data.

Figure 6 shows the number of blackouts between the same time interval for the 34 years' data. This shows a large increase in the number of blackouts from 1988 to 2009, which indicates that the number of blackouts in the China power grid has an upward trend.

Figure 7 shows the load loss of the time interval of blackout size for the 34 years' data. This shows a large increase in the load loss of the time interval of blackout size from 1981 to 2009, which indicates that the load loss of the time interval of blackout size in the China power grid has an upward trend. 


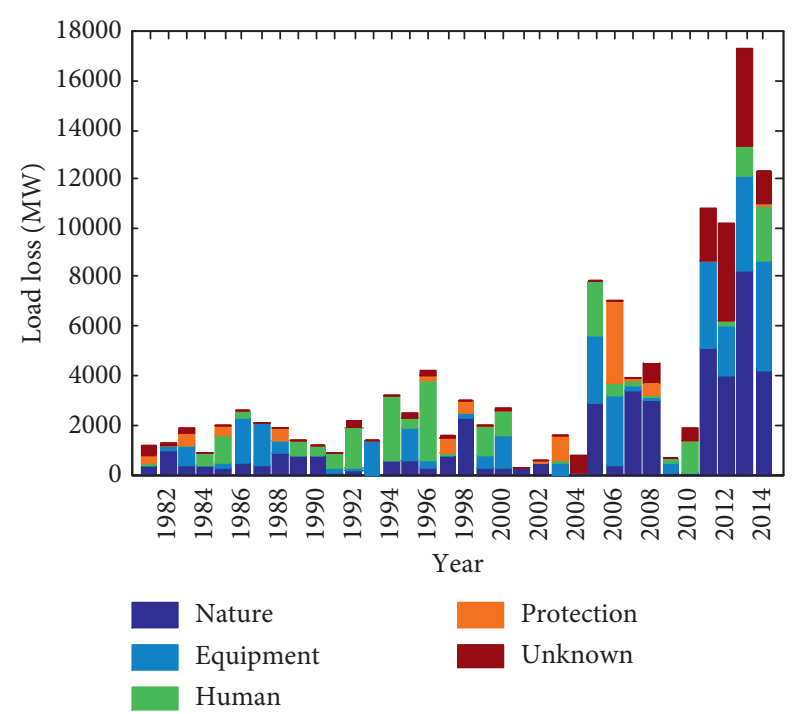

Figure 5: The load loss of blackout size per year in the China power grid.

TABLE 3: Statistics of the time interval of blackout data in the China power grid.

\begin{tabular}{lccc}
\hline $\begin{array}{l}\text { Time } \\
\text { interval }\end{array}$ & $\begin{array}{c}\text { Blackout } \\
\text { frequency }\end{array}$ & $\begin{array}{c}\text { Load loss } \\
(\mathrm{MW})\end{array}$ & $\begin{array}{c}\text { Mean size in each } \\
\text { blackout (MW) }\end{array}$ \\
\hline $1981-1987$ & 56 & $11,833.5$ & 211.3 \\
$1988-1994$ & 32 & $12,065.9$ & 377.1 \\
$1995-2001$ & 49 & $16,169.2$ & 330.0 \\
$2002-2008$ & 72 & 26,412 & 366.9 \\
$2009-2014$ & 68 & 53,108 & 781.0 \\
\hline
\end{tabular}

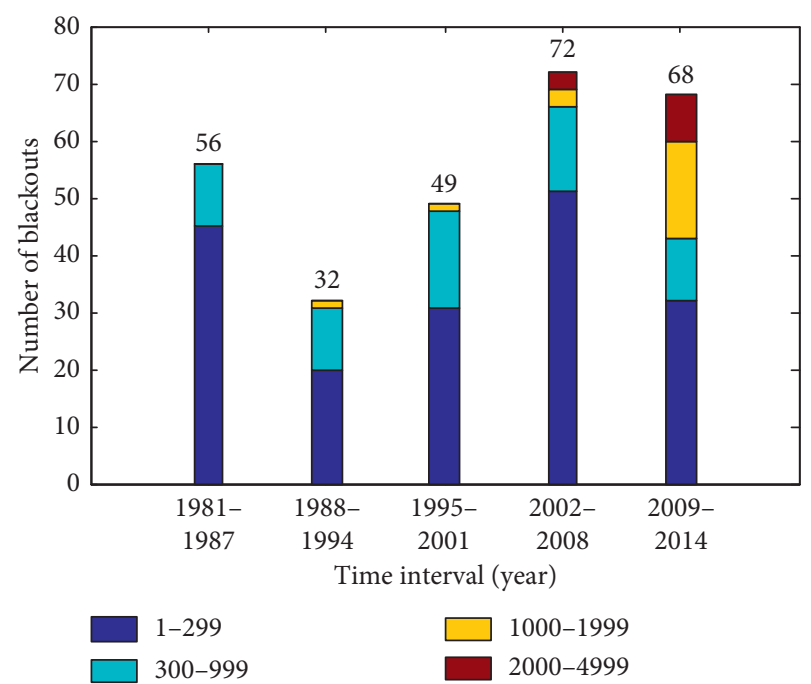

FIgURE 6: The number of blackouts between the same time interval in the China power grid.

Figures 6 and 7 show that, in recent years, the number of blackouts and load loss of the time interval of blackout size in the China power grid show an upward trend but have not

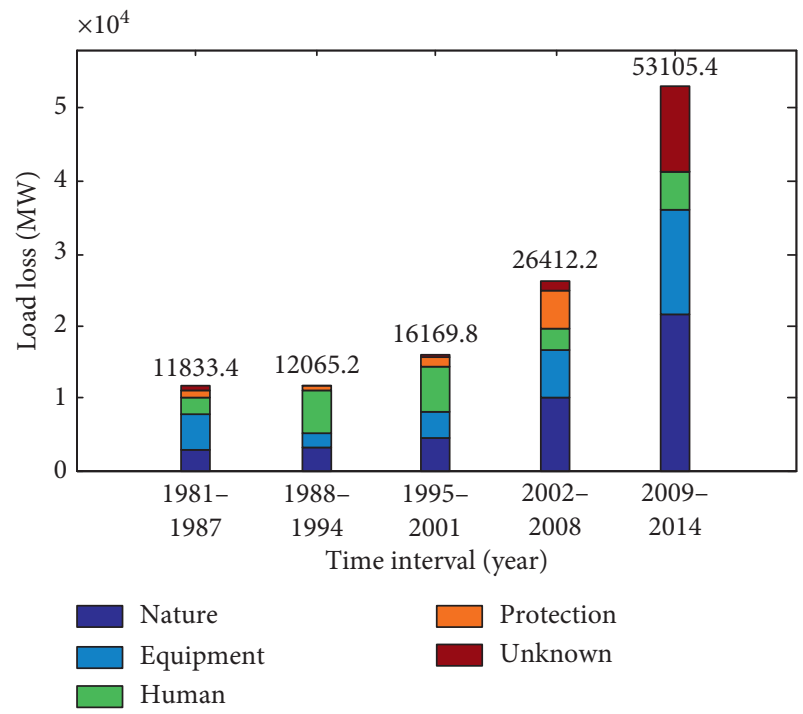

FIgURE 7: The load loss of blackouts between the same time interval in the China power grid.

decreased with time; this confirms our analysis that blackout frequency and blackout size in the China power grid have not decreased over time during the years from 1981 to 2014, and in fact, they have an upward trend.

4.2. Seasonal Trends of the Blackout. Figures 8 and 9 show the seasonal trend of blackout frequency. The peak value of summer months is obvious, and statistical analysis supports this view. The average number of monthly blackouts is 23.08 times, and the $95 \%$ confidence interval is 19.15 to 27.01 . Blackouts in July and August are 32 and 31, respectively, well beyond these ranges, indicating that the monthly data do not match the same distribution, so the seasonal trend of the number of blackouts is present. In addition, we also find the highest number of blackouts in summer followed by spring, and the highest number of blackouts occurred in July followed by August.

Figures 10 and 11 show the seasonal trend of blackout size. The peak value of summer months is obvious, and statistical analysis supports this view. The average load loss of monthly blackouts is $9,965.8 \mathrm{MW}$, and the $95 \%$ confidence interval is $6,977 \mathrm{MW}$ to $12,955 \mathrm{MW}$. The load loss of blackout size in July and May is 19,816.9 MW and 15,552.3 MW, respectively, well beyond these ranges, indicating that the monthly data did not match the same distribution, so the seasonal trend of the load loss of blackout size is present. In addition, we also find the highest load loss of blackout size in summer followed by spring, and the highest number of blackouts occurred in July followed by May.

By statistical analysis, we find that blackout frequency and blackout size show an obvious seasonal trend. As can be seen from Figures 8-11, blackout frequency and blackout size increase significantly during the summer and spring months, so we can reject this hypothesis and conclude that 


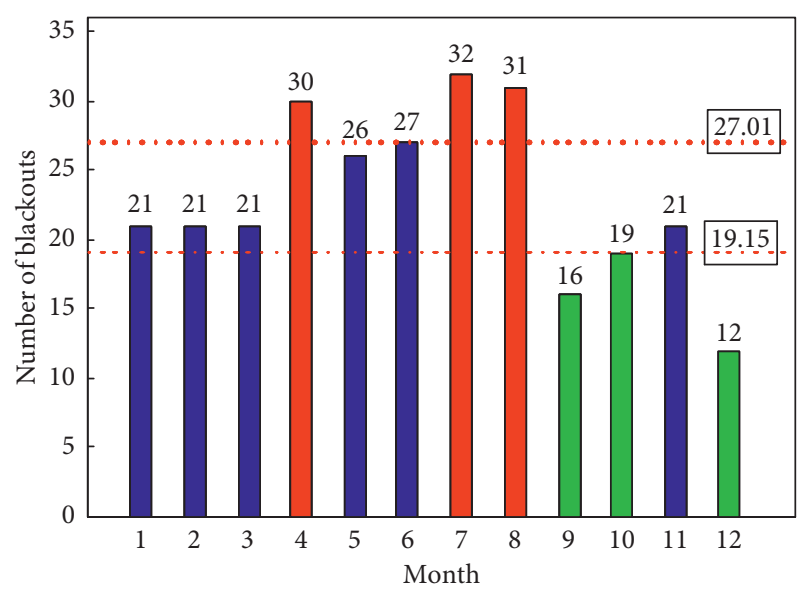

Figure 8: The number of blackouts every month in the China power grid.

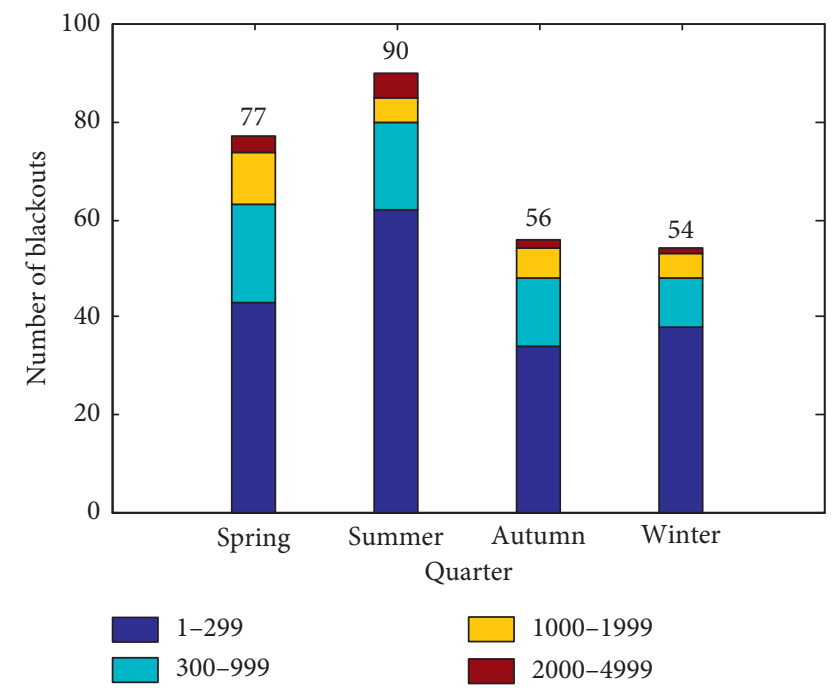

FIgURE 9: The number of blackouts in each quarter in the China power grid.

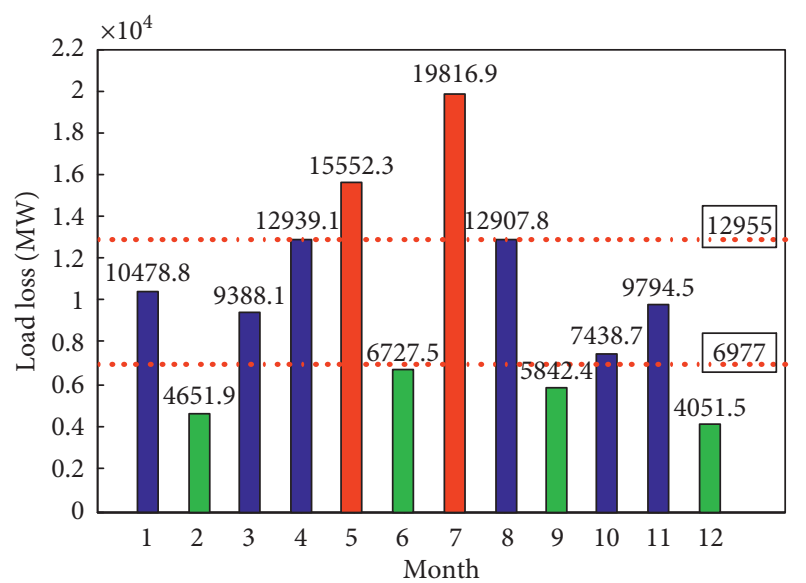

Figure 10: The load loss of blackout size every month in the China power grid.

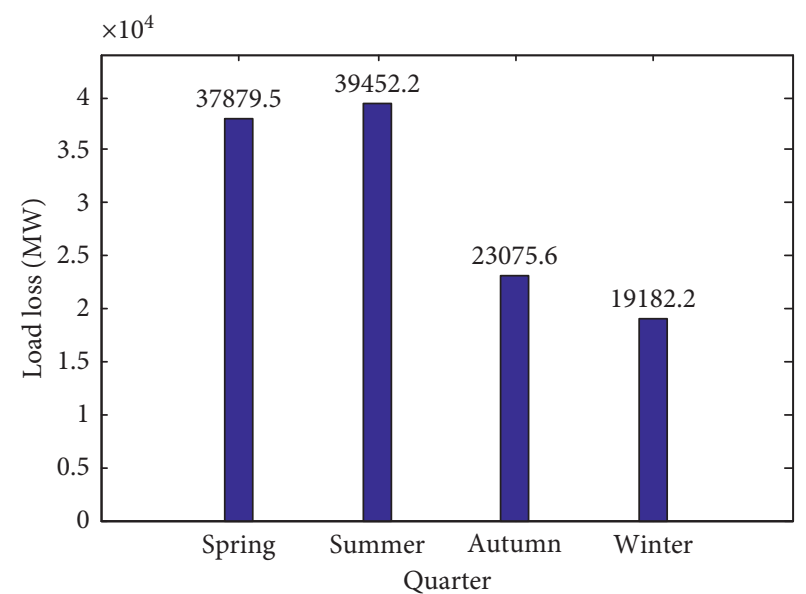

FIgure 11: The load loss of blackout size in each quarter in the China power grid.

the risk of blackouts does change over time. Besides, we also find that blackout frequency and blackout size which occurred in summer are the largest followed by spring. In July, blackout frequency and blackout size are the largest.

\section{Conclusion}

This paper presents the method of $V / S$ to calculate the longrange correlation of blackouts in the China power grid. This paper also proposed a rigorous method to analyze the power-law distribution of blackouts. The simulation results verify that the time series of blackouts in the China power grid has obvious self-organized criticality. In addition, we use mathematical statistics to analyze the possible trend of blackout data in the China power grid and provide more rigorous analysis by using appropriate statistical tests. The conclusions can be summarized as follows:

(1) The SOC of blackouts in the China power grid indicates that blackouts may be inevitable, and large blackouts are rare but expected to occur occasionally. The blackout frequency and blackout size in the China power grid have not decreased over time, and they have the possibility of an upward trend, indicating that blackout risk does not decrease over time. This is true, although power companies have put a lot of effort in improving the security and reliability of their power systems.

(2) The seasonal trend of blackouts in the China power grid indicates that the risk of blackouts does change over time. This conclusion may guide the electricity industry to formulate a time maintenance plan to improve grid reliability. For example, in summer of the highest risk, we can increase the number of operator staff on duty and the number of overhauls of the power grid, thus reducing the risk of blackouts.

\section{Data Availability}

The data, provided in the article, were collected from a report on the safety generation accident of National Power Grid 
Corp and China Electric Power Press. They are available in the public domain, so the authors have no restriction on the data.

\section{Conflicts of Interest}

The authors declare that they have no conflicts of interest.

\section{Acknowledgments}

This work was supported by the Science and Technology Project of SGCC in 2018 (Multisand Pile Theory of Interconnection Power Grid Blackouts Early Warning Technology and System Development, no. XTB17201800166).

\section{References}

[1] J. B. Guo, Y. H. Yin, and G. C. Yao, "Statistic and analysis for instability incidents in the bulk of power systems in 1998-1991," Power System Technology, vol. 18, no. 2, pp. 58-61, 1994.

[2] J. B. Guo, "Statistics and analysis of incidents in bulk power system from 1991 to 1995," Power System Technology, vol. 22, no. 2, pp. 72-74, 1998.

[3] J. Qu and J. B. Guo, "Statistics and analysis of faults in main domestic power system from 1996 to 2000," Power System Technology, vol. 28, no. 21, pp. 60-63, 2004.

[4] Q. Yu and J. B. Guo, "Self-organized criticality and its application in power system collapse prevention," in Proceedings of the 2006 International Conference on Power System Technology, pp. 1-3, IEEE Computer Society, New York, NY, USA, 2006.

[5] Q. Yu and J. B. Guo, "Statistics and self-organized criticality characters of blackouts in China Electric Power Systems," Automation of Electric Power Systems, vol. 30, no. 2, pp. 16-21, 2006.

[6] Q. Yu and J. B. Guo, "Study on self-organized criticality of power system blackouts in China," Power System Technology, vol. 30, no. 6, pp. 1-5, 2006.

[7] Q. Yu and J. B. Guo, "Self-organized criticality and extreme statistics analysis of electric power system blackouts," Automation of Electric Power Systems, vol. 31, no. 3, pp. 1-3, 2007.

[8] X. Duan and S. Su, "Self-organized criticality in time series of power systems fault, its mechanism, and potential application," IEEE Transactions on Power Systems, vol. 25, no. 4, pp. 1857-1864, 2010.

[9] D. F. Chao, J. X. Yang, S. Su, and D. Y. Shi, "Self-organized criticality and its cause of faults in Puyang distribution system," Power System Technology, vol. 35, no. 1, pp. 72-75, 2011.

[10] B. B. Mandelbrot and J. R. Wallis, "Noah, joseph, and operational hydrology," Water Resources Research, vol. 4, no. 5, pp. 909-918, 1968.

[11] L. X. Xu, J. M. Yang, Z. Chao, and M. B. Liu, "Statistical distribution and nonlinear characteristic analysis of fault time intervals in power grids," Power System Technology, vol. 37, no. 12, pp. 3482-3488, 2013.

[12] Q. Yu, Y. Q. Qu, and L. Shi, "Self-correlation analysis of power grid blackouts based on relative value method and Hurst exponent," Automation of Electric Power Systems, vol. 42, no. 1, pp. 55-60, 2018.

[13] Q. Yu, Y. Q. Qu, N. Cao, and J. Yi, "Statistics and distribution characteristics analysis of time interval of blackout in Chinese power grid," SCIENTIA SINICA Technologica, vol. 49, no. 1, pp. 55-62, 2018.

[14] B. A. Carreras, D. E. Newman, I. Dobson, and A. B. Poole, "Initial evidence for self-organized criticality in electric power system blackouts," in Proceedings of the the 33rd Annual Hawaii International Conference on System Sciences, IEEE Computer Society, New York, NY, USA, pp. 1-6, 2000.

[15] B. A. Carreras, D. E. Newman, I. Dobson, and A. B. Poole, "Evidence for self-organized criticality in a time series of electric power system blackouts," IEEE Transactions on Circuits and Systems I: Regular Papers, vol. 51, no. 9, pp. 17331740, 2004.

[16] P. Bak, C. Tang, and K. Wiesenfeld, "Self-organized criticality: an explanation of $1 / f$ noise," Physical Review Letters, vol. 59, no. 4, pp. 381-384, 1988.

[17] J. S. Simonoff, C. E. Restrepo, and R. Zimmerman, "Riskmanagement and risk-analysis-based decision tools for attacks on electric power," Risk Analysis, vol. 27, no. 3, pp. 547-570, 2007.

[18] M. Amin, "Energy infrastructure defense systems," Proceedings of the IEEE, vol. 93, no. 5, pp. 861-875, 2007.

[19] P. Hines, J. Apt, and S. Talukdar, "Large blackouts in North America: historical trends and policy implications," Energy Policy, vol. 37, no. 12, pp. 5249-5259, 2009.

[20] R. Weron and I. Simonsen, "Blackouts, risk, and fat-tailed distributions," in Proceedings of the Third Nikkei Econophysics Symposium, Springer Science and Technology, Tokyo, Japan, pp. 215-219, 2004.

[21] D. Cornforth, "Long tails from the distribution of 23 years of electrical disturbance data," in Proceedings of the 2009 IEEE/ PES Power Systems Conference and Exposition, pp. 1-8, IEEE Computer Society, New York, NY, USA, 2009.

[22] B. A. Carreras, D. E. Newman, and I. Dobson, "North American blackout time series statistics and implications for blackout risk," IEEE Transactions on Power Systems, vol. 31, no. 6, pp. 4406-4414, 2016.

[23] Q. Yu, "Research on self-organized critical characteristics of power system blackouts," Doctoral dissertation, China Electric Power Research Institute, Beijing, China, 2010.

[24] National Electric Power Dispatching and Communication Center, Analysis of Typical Accidents of National Power Grid (1988-1998), China Electric Power Press, Beijing, China, 2000.

[25] National Electric Power Dispatching and Communication Center, Analysis of Typical Accidents of National Power Grid (1999-2007), China Electric Power Press, Beijing, China, 2008.

[26] National Power Grid Corp Security Supervision Department, Report on the Safety Generation Accident of the National Power Grid Corp in 2008, China Electric Power Press, Beijing, China, 2009.

[27] National Power Grid Corp Security Supervision department, Report on the Safety Generation Accident of the National Power Grid Corp in 2009, China Electric Power Press, Beijing, China, 2010.

[28] National Power Grid Corp security supervision department, Report on the Safety Generation Accident of the National Power Grid Corp in 2010, China Electric Power Press, Beijing, 2011.

[29] National Power Grid Corp Security Supervision department, Report on the Safety Generation Accident of the National Power Grid Corp in 2011, China Electric Power Press, Beijing, China, 2012.

[30] National Power Grid Corp Security Supervision Department, Report on the Safety Generation Accident of the National Power Grid Corp in 2012, China Electric Power Press, Beijing, China, 2013. 
[31] National Power Grid Corp Security Supervision Department, Report on the Safety Generation Accident of the National Power Grid Corp in 2013, China Electric Power Press, Beijing, 2014.

[32] National Power Grid Corp Security Supervision Department, Report on the Safety Generation Accident of the National Power Grid Corp in 2014, China Electric Power Press, Beijing, China, 2015.

[33] A. Clauset, C. R. Shalizi, and M. E. J. Newman, "Power-law distributions in empirical data," Siam Review, vol. 51, no. 4, pp. 661-703, 2009.

[34] H. E. Hurst, "Long-term storage capacity of reservoirs," American Society of Civil Engineers, vol. 116, no. 12, pp. 770-799, 1951.

[35] L. Giraitis, P. Kokoszka, R. Leipus, and G. Teyssière, "Rescaled variance and related tests for long memory in volatility and levels," Journal of Econometrics, vol. 112, no. 2, pp. 265-294, 2003. 